\title{
Atypical Features in Regenerating Tubules Point to a Risk for Implantation of Renal Stem/Progenitor Cells
}

Review Article

Minuth $\mathrm{WW}^{*}$, Denk L

Department of Molecular and Cellular Anatomy, University of Regensburg, Germany.

\section{Abstract}

Stem/progenitor cells are seen as a therapeutic option for repair of diseased renal parenchyma. However, actual data show that survival of implanted stem/progenitor cells is impeded by harmful interstitial environment.

To learn about parameters for cell adaptation, renal stem/progenitor cells were mounted in a polyester (POSI-4) interstitium during perfusion culture. Controlled fluid environment was maintained by chemically defined $\mathrm{CO}_{2}$ - independent culture media for 13 days. Cell biological features were then analyzed by immunohistochemistry, while structural details were investigated by advanced fixation of specimens for microscopy. When stem/progenitor cells are kept in Leibovitz's L-15 Medium or $\mathrm{CO}_{2}$ Independent Medium, spatial development of numerous tubules is observed. Immunolabel for TROMAIII, cingulin and laminin $\gamma 1$ depicts that a homogenous cell population is contained. Semithin sections of specimens fixed in traditional glutaraldehyde (GA) solution reflect an unobtrusive morphology. In contrast, fixation by GA solution including ruthenium red unveils in tubules a thickened basal lamina. Fixation by GA solution including tannic acid illustrates atypical development of a heterogeneous epithelium consisting of bright and dark cells.

Thus, advanced fixation of specimens makes pathological features visible, when regeneration is investigated by renal stem/ progenitor cells. To what extent a comparable risk lurks behind an implantation, has to be elaborated.

Keywords: Kidney; Stem/Progenitor Cells; Niche; Regeneration; Artificial Interstitium; Polyester Fleece; Perfusion Culture; Chemically Defined Culture Medium.

\section{*Corresponding Author:}

Will W. Minuth,

Department of Molecular and Cellular Anatomy, University of Regensburg, University Street 31, D-93053 Regensburg, Germany.

Fax: +499419432868

Email: will.minuth@vkl.uni-regensburg.de

Received: May 27, 2015

Accepted: June 22, 2015

Published: June 24, 2015

Citation: Minuth WW, Denk L (2015) Atypical Features in Regenerating Tubules Point to a Risk for Implantation of Renal Stem/Progenitor Cells. Int J Stem Cell Res Transplant 03(2), 101-108. doi: http://dx.doi. org $/ 10.19070 / 2328-3548-1500017$

Copyright: Minuth $\mathbf{W W}^{\circ}$ 2015. This is an open-access article distributed under the terms of the Creative Commons Attribution License, which permits unrestricted use, distribution and reproduction in any medium, provided the original author and source are credited.

\section{Introduction}

The repair of diseased renal parenchyma initiated by the implantation of stem/progenitor cells is seen since years as an innovative therapeutic challenge [1-4]. However, critical analysis of actual publications demonstrates that a real breakthrough was up to date not achieved and the project still drifts more in a phase of preclinical research than in successful clinical trials.

\section{Switch from comfortable to harmful Environment}

Recent literature informs that stem/progenitor cells can be principally administered via the arterial or venous vessel system, by punctual injections into diseased renal parenchyma or by seeding in the space left between the organ capsule and the outer cortex [5-7]. Although various sites for implantation were tested, reasons for an only minimal survival of implanted cells are unclear [8]. A general impression is that the implantation starts and ends by a simple injection of selected stem/progenitor cells. However, in this coherence it is not considered that the application of cells at the moment of implantation is only the inducing link, which belongs to a complex biomedical chain leading hopefully to regeneration of diseased renal parenchyma. To shed some light in the small degree of stem/progenitor cell survival in diseased renal parenchyma, analytical focus must be directed to the original environment of stem/progenitor cells in comparison to the procedure of implantation and successive seeding. Fact is that in embryonic renal parenchyma epithelial and mesenchymal stem/ progenitor cells occur within the special environment of a highly structured niche [9]. Further both types of stem/progenitor cells are separated by a special interstitial interface containing abundant but filigree extracellular matrix [10-12]. Despite separation both types of stem/progenitor cells are not functionally isolated, but 
connected via tunneling nanotubes $[13,14]$.

Further on, independent on whether renal or other types of stem/ progenitor cells are under consideration for implantation, they must be isolated and then incubated in a culture medium supporting development before an injection is made $[15,16]$. When implantation is performed, one has to realize that a co-implanted culture medium immediately dilutes so that stem/progenitor cells are surrounded from one second to the other by diseased renal parenchyma. Here they are exposed to degenerating epithelia, altered extracellular matrix, unbalanced electrolytes, growth factors, interleukins and hormones. It is obvious that such an environment does not stimulate intended repair [17-20]. As a result, the majority of cells is damaged so that only a small fraction will be able to migrate to the site of restoration. But how can repair of diseased parenchyma be realized, when stimulating interstitial fluid and attractive extracellular matrix are lacking? In this situation it appears that implanted stem/progenitor cells are overstrained and that one expects more than they can really do.

\section{Buffering environment facilitates the transitional period}

When stem/progenitor cells are implanted in diseased renal parenchyma, the main problem is that they are exposed from one second to the other to a stressing environment. Thus, the concept for an adaption is to create a buffering environment and to coimplant it with stem/progenitor cells. The technical answer is to seed selected cells in an artificial interstitium and to implant the ensemble as it was earlier proposed [21]. In this scenario stem/ progenitor cells are kept in a polyester fleece. Comparable to the original niche the fibers of the fleece mimic extracellular matrix, while the space between acts as a reservoir for fluid. In a first step a fleece with included cells can be inserted in a perfusion culture container. Here cells are optimally provided with nutrition and respiratory gas by the transport of always fresh medium. Stem/ progenitor cells seeding in an artificial interstitium can be used for advanced in vitro experiments necessarily needed in preclinical research (Figure 1a-c). For example, promoting or inhibiting effects of drugs on developmental capacity of contained stem/ progenitor cells can be tested yet adapted to realistic conditions. When fiber coating, fluid composition, morphogenetic factors, anti-inflammatory drugs or respiratory gas are modulated, essential information about capacity for repair will be collected. Finally, when satisfactory data are generated and an implantation comes in sight as a second step, an optimized fleece loaded first with stem/progenitor cells can be implanted by minimal invasive tools in the space between the renal organ capsule and the outer parenchyma (Figure 1d-f).

However, the presented idea, concept and reality must be brought to concurrence. Yet, more than 10 years of practical experiences show that the search for a suitable fleece as artificial interstitium and a stable culture medium for implantation takes more time than it was earlier anticipated. For this reason actual research in our laboratory is still in a preclinical phase dealing with advanced in vitro experiments (Figure 1a-c). To show sustainable success of this strategy but also to give insights in new problems, some of actual results are presented.

\section{Isolation of renal niches}

When developmental capacity is tested, renal stem/progenitor cell niches must be easily preparable and they must be available in sufficient quality and reproducible quantity for cell biological

Figure 1. Schema illustrates seeding of renal stem/progenitor cells in an artificial interstitium for (a-c) preclinical research and (d-f) clinical application. (a) Stem/progenitor cells (s/pC) are isolated by stripping off the capsule of neonatal rabbit kidney with fine forceps. (b) An artificial interstitium is created by placing isolated tissue between layers of a POSI-4 polyester fleece (PF) within a Minusheet ${ }^{\circledR}$ tissue carrier. (c) The carrier is transferred to a perfusion culture container with horizontal flow characteristics. Then culture is performed for 13 days at $37^{\circ} \mathrm{C}$ under atmospheric air. During this period always fresh chemically defined culture medium is transported by a peristaltic pump $(1.25 \mathrm{ml} / \mathrm{h})$ from a storage bottle to the container and then to a waste bottle. (d) For an intended implantation cells are loaded in a fleece with integrated tubes (arrows). (e) Then a fleece pad with integrated tubes is implanted under the organ capsule und connected with other fleeces. (f) After implantation fleeces are perfused with always fresh culture medium and respiratory gas to protect contained cells against harsh surrounding of diseased parenchyma. At an afferent tube medication can be given, while on an efferent tube physiological parameters are registered.

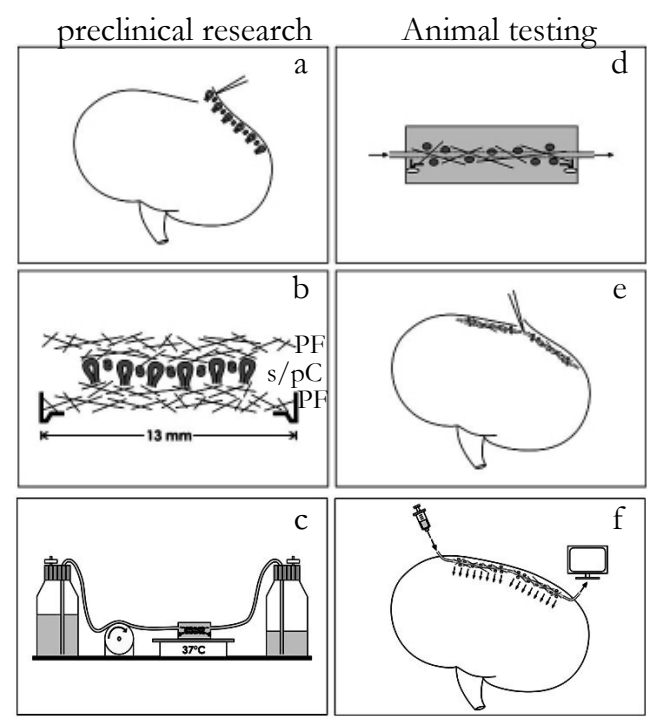


analysis. For current research it was decided to use kidneys derived from neonatal rabbit. To the best of our knowledge, up to date no other species is known, which makes it possible to harvest renal niches in shortest time, in such an amount and quality.

Briefly, for present experiments kidneys from one-day old New Zealand rabbits (Seidl, Oberndorf, Germany) were isolated under sterile conditions and cut into a ventral and dorsal half as earlier described [22]. Then the fibrous organ capsule was stripped off by fine forceps to obtain a constantly thin layer of stem/progenitor cell niches adherent to the explant (Figure 1a). Applying this method embryonic tissue of up to $1 \mathrm{~cm}$ in square can be isolated for use in culture experiments and morphological analysis.

\section{Artificial interstitium for in vitro experiments}

For present experiments renal stem/progenitor cell niches were isolated to investigate developmental capacity and spatial formation of tubules. Due to suitable stimuli it is obvious that such experiments cannot be performed in a simple dish. To facilitate spatial development, a common technique is to coat stem/progenitor cells by proteins derived from extracellular matrix (ECM). However, personal experiences revealed that during culture the coat of ECM first supports but then hinders development of tubules. This is caused by the aspic like consistency of ECM coat leading to unstirred layers of fluid. This effect accumulates harmful metabolites and hinders provision with fresh nutrition and respiratory gas. To solve the problem, isolated renal stem/progenitor niches are mounted in a polyester fleece serving as an artificial interstitium as it was earlier described [22]. Application of this technique leads between fibers of the fleece to an even distribution of fluid preventing in turn pressure peaks.

To prepare an artificial interstitium, isolated tissue containing renal stem/progenitor cell niches (Figure 1a) is placed between punched out layers $(13 \mathrm{~mm}$ in diameter) of a polyester fleece. In earlier investigations an 17 fleece (Walraf, Grevenbroich, Germany) or POSI-4, POSI-5, POSI-6 and POSI-7 fleeces (Positech, Hallwil, Suisse) were successfully applied [21, 22]. To obtain more information about density of generated tubules, in the presented experiments isolated renal stem/progenitor cell niches were mounted between layers of a POSI-4 fleece (Figure 1b). To keep the sandwich in position, it is transferred to a Minusheet ${ }^{\mathbb{R}}$ tissue carrier (Minucells and Minutissue, Bad Abbach, Germany) and then inserted in a perfusion culture container with horizontal flow characteristics (Figure 1c). To provide nutrition and respiratory gas for a period of 13 days, always fresh culture medium is continuously transported at a rate of $1.25 \mathrm{ml} / \mathrm{h}$ with an IPC N8 peristaltic pump (Ismatec, Wertheim, Germany). Further on, all of the culture experiments are performed under atmospheric air on a thermo plate (Medax-Nagel, Kiel, Germany) at $37^{\circ} \mathrm{C}$.

Under described experimental conditions up to date unsolved biomedical problems can be adequately investigated. For example, of special interest is to increase developmental capacity of stem/progenitor cells seeding them in new types of biodegradable fleeces impregnated with anti-inflammatory drugs or morphogenetic proteins (Figure 1c). A further topic is to stimulate physiological tolerance and to learn about cell adaption, when rich culture environment is switched over to a simulated harmful interstitial fluid. Thus under realistic conditions can be investigated, when implanted stem/progenitor cells come in contact with dis- eased renal parenchyma [23-28]. It is obvious that developmental capacity of implanted stem/progenitor cells must be reinforced to compensate stressing environmental parameters. The challenge for the future is consequently to learn about stimuli supporting stemness, proliferation, differentiation and spatial development although restoration in harmful environment must take place.

\section{Application of an Artificial Interstitium for Im- plantation}

When sound knowledge is available from the preclinical area (Figure 1a-c), in a second phase the concept of seeding stem/ progenitor cells in an artificial interstitium could be taken over for testing in animals (Figire 1d-e). The goal is to elaborate an effective implantation technique for stem/progenitor cells that acts as a buffering fence. It must protect on the one hand implanted stem/progenitor cells against destructive molecules occurring in diseased parenchyma and must support on the other hand initial seeding, proliferation, migration, differentiation and subsequent spatial repair after an implantation.

The technical concept is to seed stem/progenitor cells in a polyester fleece serving as an artificial interstitium [21,22]. However, in this scenario additionally an afferent and an efferent tube is integrated within the fleece pad so that contained stem/progenitor cells can be provided before, during and after transplantation with always fresh culture medium (Figure 1d). This implantation technique produces a constant environment and a stable $\mathrm{pH}$ over a prolonged period of time.

For a successful implantation it is further intended to insert several fleece pads in the space between the renal organ capsule and outer cortex of parenchyma (Figure 1e). Since tubes are integrated, implanted fleece pads can be connected with each other. This way all of contained cells are provided with nutrition and respiratory gas during the initial phase of implantation. Finally, medium that is transported in an efferent tube can be analyzed so that parameters about the actual situation within an implanted pad are transparent (Figure 1f). Physiological data will reflect optimal repair, while un-physiological measures signal that a correction by individual medication has to be made.

\section{Need for chemically defined culture media}

Although frequently not considered, in most of the cases presently used culture media for stem/progenitor cell research contain serum, other undefined additives and a buffer system based on $\mathrm{NaHCO}_{3}$. However, regarding a most probable metabolic alkalosis in diseased renal interstitium, uncontrolled influence of serum on development and finally avoiding any risk of infection, only chemically defined culture media are under current use in our laboratory.

Since a culture medium is essential for implantation, it must be guaranteed that its $\mathrm{pH}$ stays stable independent whether it is used in an incubator, under atmospheric air or after implantation in the avascular environment of diseased renal parenchyma. Further on, isolation, cell mounting in a fleece, subsequent perfusion culture and implantation are carried out under atmospheric air in the laboratory. Due to this reason a culture medium must be selected that lacks respectively contains only low $\mathrm{NaHCO}_{3}$ due to low $\mathrm{CO}_{2}$ 
concentration in atmospheric air.

As a consequence, for present experiments chemically defined Leibovitz's L-15 Medium (Nr. 31415-029) and $\mathrm{CO}_{2}$ Independent Medium (Nr. 18045-054) both including Phenolred (GIBCO/Invitrogen, Karlsruhe, Germany) are selected for use in the laboratory $[29,30]$. Infections are prevented by adding an antibiotic-antimycotic cocktail (1\%, GIBCO/Invitrogen). As earlier described, formation of tubules is induced by administration of aldosterone (1 x $10^{-7} \mathrm{M}$, Fluka, Taufkirchen, Germany) [21, 22]. In described culture experiments a constant $\mathrm{pH}$ of 7.4 is maintained by $\mathrm{CO}_{2}$ Independent Medium, while $\mathrm{pH}$ in Leibovitz's L-15 Medium has to be adjusted by adding aliquots of $\mathrm{N}-2$-HydroxyethylpiperazineN-2-Ethane Sulfonic Acid (HEPES; GIBCO/Invitrogen).

\section{Unspectacular morphological features of a poly- ester fleece}

An artificial interstitium must promote spatial development of stem/progenitor cells. Excellent experiences were made by a fleece consisting of woven respectively compressed fibers mimicking extracellular matrix (ECM). For present experiments a POSI-4 polyester fleece was selected. It has a thickness of 0.13 $[\mathrm{mm}]$, a water porosity of $1480\left[\mathrm{~L} / \mathrm{m}^{2} / \mathrm{S}\right]$ and a micron rating of $40[\mu \mathrm{m}]$. Due to its small thickness it is possible to increase space for spatial development by the use of 4 layers POSI-4 fleece for cell seeding instead of a single layer of an earlier applied $\mathrm{I} 7$ fleece $(0.6 \mathrm{~mm})$ [21]. By piling and paving the extension of the fleece and thereby the density of regenerating tubules can be influenced [31].

Of particular interest is the surface and orientation of polyester fibers in an applied fleece. It is supposed that these parameters influence spatial formation of tubules. To analyze those features in detail, scanning electron microscopy must be performed. As an example, for optimal results a POSI-4 polyester fleece is sputtercoated with gold (Polaron E 5100, Watford, GB) and analyzed in a DSM 940A scanning electron microscope (Zeiss, Oberkochen,
Germany) (Figure 2a). Images of the screen are taken by a Pentax SLR Digital Camera and processed with Adobe Photoshop 7.0 (Adobe, California, USA) and Corel DRAW Suite X5 (Corel Corporation, Otawa, Canada). An analyzed polyester fleece demonstrates that its fibers are lining in a longitudinal, transversal and oblique course. They show a homogenous composition and a surprisingly smooth surface without any recognizable protrusions or roughness. The average diameter of a fiber is $10 \mu \mathrm{m}$. When they are crossing, the contact site appears slightly dilated but does not exhibit any remarkable signs. Thus, the orientation and distribution of all fibers are fully inconspicuous. In each case the surface of a fiber does not explain their hidden capacity to promote spatial development of renal tubules in combination with a chemically defined culture medium.

\section{Immunohistochemistry suggests intact develop- ment}

When stem/progenitor cells are seeding in an artificial interstitium, development depends on both an intrinsic cell biological capacity and the environment reflected by fiber composition of the applied fleece and/or composition of the culture medium. To obtain concrete information about inhibiting or promoting influences, in the present set of experiments renal stem/progenitor cells were mounted in a POSI-4 fleece (Figure 2a) and cultured in combination with chemically defined $\mathrm{CO}_{2}$ Independent Medium (Figure 2b) or Leibovitz's L-15 Medium (Figure 2c). Of main interest was to register, if an even distribution of generated tubules within the fleece takes place. For this analysis a quick and reliable technique for exact visualization is needed. Excellent experiences were made by whole mount immunohistochemistry on generated tubules in combination with confocal laser scanning microscopy (Figure 2b,c).

A reliable method is to fix a POSI-4 fleece with adherent renal tubules in $70 \%$ ethanol. Before labeling specimens are washed several times with phosphate buffered saline (PBS, pH 7.5) and

Figure 2. Scanning electron microscopy (SEM) of a POSI-4 polyester fleece and confocal fluorescence microscopy of tubules showing spatial growth between the fleece fibers. (a) SEM shows an unobtrusively looking surface of polyester fibers

(PF). Whole mount label for cytokeratin 19 (TROMA-III) illustrates under low magnification development of numerous tubules ( $T$ ), when specimens are raised in (b) $\mathrm{CO}_{2}$ Independent Medium or (c) Leibovitz's L-15 Medium.

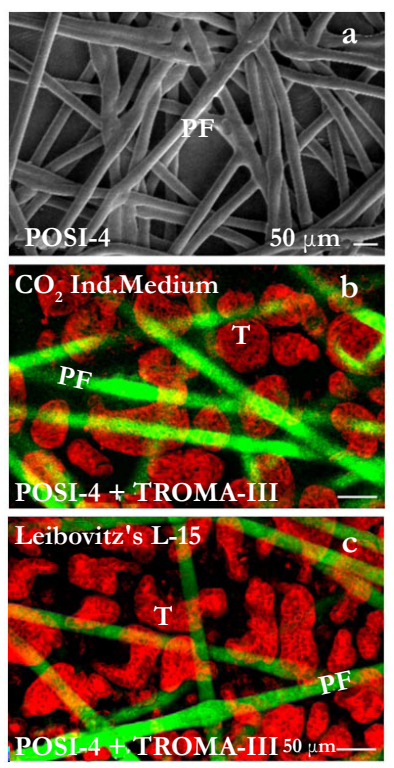


incubated for 30 minutes with blocking solution (PBS, 10\% horse serum from GIBCO/Invitrogen, $1 \%$ bovine serum albumin from Serva, Heidelberg, Germany). Then an antibody such as anti-cytokeratin 19 (TROMA-III, Developmental Studies Hybridoma Bank DSHB, Iowa, USA), anti-cingulin (Progen Biotechnik, Heidelberg, Germany) or anti-laminin $\gamma 1$ (clone 3610, Santa Cruz Biotechnology, Santa Cruz, USA) is applied for one hour. After washing with $1 \%$ BSA in PBS the specimens are incubated for 45 minutes with goat-anti-rat-IgG-rhodamine or donkey-anti-guinea-pig-IgG-fluorescein-isothiocyanate (Jackson Immunoresearch Laboratories, West Grove, USA) diluted 1:50 in PBS containing $1 \%$ BSA. Following several washes with PBS labeled specimens are analyzed using a CM12 confocal laser scanning microscope (Zeiss, Oberkochen, Germany). Fluorescence images are taken with a digital camera at a standard exposure time and thereafter processed with Corel DRAW Graphic Suite X5 (Corel Corporation, Otawa, Canada).

Intense whole mount label for TROMA-III exhibits in confocal microscopy that numerous tubules are growing in a POSI-4 polyester fleece, when specimens are raised in $\mathrm{CO}_{2}$ Independent Medium (Figure 2b) or Leibovitz's L-15 Medium (Figure 2c). In these cases can be seen that tubules are evenly distributed in the space between the fleece fibers. Further it is recognized that in tubules a homogenously composed epithelium with isoprismatic cells is contained (Figure 2b,c).

On selected cross and longitudinal sections TROMA-III label indicates that tubule cells are bordered by a lumen and that their basal aspect is covered by a basal lamina (Figure 3a,b). Further intense label for cingulin indicates that epithelial cells within generated tubules are polarized and exhibit a tight junction complex between the luminal and lateral plasma membranes (Figure 3c,d). Finally, label for laminin $\gamma 1$ illustrates that in series raised in both $\mathrm{CO}_{2}$ Independent Medium (Figure 3e) and Leibovitz's L-15 Medium (Figure $3 \mathrm{f}$ ) generated tubules are enclosed by an intensely marked basal lamina. Looking over all, the impression is created that generated tubules are evenly distributed and that they are well developed. It appears that there is no more risk and nothing is standing principally in the way of an earlier mentioned implanta- tion for example in an adult rabbit (Figure 1d-f).

\section{Special fixation unveils unexpected morphologi-} cal details

When morphological analysis is performed in the area of stem/ progenitor cell research, a common method is to fix specimens in glutaraldehyde (GA) solution for light and transmission electron microscopy combined with semi- and ultrathin sectioning. However, actual investigations illustrate that this traditional kind of fixation does not display all contained morphological details. To unveil such hidden structures improved fixation by GA solution including ruthenium red or tannic acid must be applied [32].

To illustrate earlier not visible pathological details, polyester fleeces containing generated tubules were harvested after 13 days of culture (Figure 1c) and fixed in the following solutions:

1. Specimens for control: 5\% GA (Serva) solution buffered with $0.15 \mathrm{M}$ sodium cacodylate, $\mathrm{pH} 7.4$.

2. Specimens for ruthenium red contrast: 5\% GA solution buffered with $0.15 \mathrm{M}$ sodium cacodylate, $\mathrm{pH} 7.4+0.5 \%$ ruthenium red (Fluka, Taufkirchen, Germany).

3. Specimens for tannic acid contrast: 5\% GA buffered with $0.15 \mathrm{M}$ sodium cacodylate, $\mathrm{pH} 7.4+1 \%$ tannic acid (SigmaAldrich Chemie, München, Germany).

To obtain a reliable presevation of morphological details, primary fixation must be performed for 1 day at room temperature. After several washes with $0.15 \mathrm{M}$ sodium cacodylate the samples are postfixed in the same buffer but additionally containing 1\% osmium tetroxide (Science Services, München, Germany). Specimens are washed then with sodium cacodylate buffer and dehydrated in graded series of ethanols. Finally, specimens are embedded in Epon (Fluka) and polymerized at $60^{\circ} \mathrm{C}$ for $48 \mathrm{~h}$. Semithin sections are best made with a diamond knife on an ultramicrotome such as EM UC6 (Leica, Wetzlar, Germany). Analysis of semithin sections is performed with an Axioscope microscope (Zeiss). Images are finally taken with a digital camera (AxioCam MRC, Zeiss) and processed with Corel DRAW Graphic Suite X5 (Corel Corpora-

Figure 3. Confocal fluorescence microscopy of tubules regenerating in a POSI-4 polyester fleece. Specimens are raised in (a,c,e) $\mathrm{CO}_{2}$ Independent Medium and (b,d,f) Leibovitz's L-15 Medium. Whole mount label for (a,b) cytokeratin 19 (TROMA-III) depicts in both series development of numerous tubules (T). (c, d) Label for cingulin demonstrates polarization of tubule cells and formation of a tight junction complex (arrow). (e, $f$ ) Intense label for laminin $\gamma 1$ shows formation of a basal lamina (asterisk) on generated tubules.

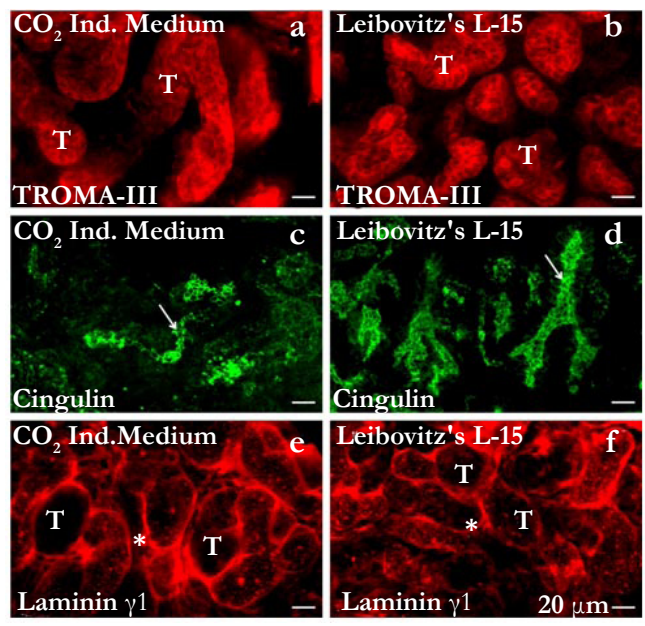


tion) as it was earlier described [33].

\section{A closer look reveals atypical cell features}

Semithin sections of specimens fixed in traditional GA solution and stained with Richardson have an unremarkable appearance and exhibit that numerous tubules are developing within the artificial interstitium (Figure 4a,b). In series with both $\mathrm{CO}_{2}$ Independent Medium (Figure 4a) and Leibovitz's L-15 Medium (Figure 4b) can be seen that a single epithelial cell layer and a homogenous population is contained in generated tubules. Dependent on the section plane it is further recognized that the luminal side of cells borders a lumen, while the basal aspect rests on a barely recognizable basal lamina. In several cases the lumen is filled with apoptotic cells and a special luminal matrix as it is known from other developing epithelia [34]. Vacuoles in the cytoplasm of generated tubule cells occasionally occur. Finally, differences in morphological quality of tubule cells cannot be recognized.

In contrast, when specimens are raised in $\mathrm{CO}_{2}$ Independent $\mathrm{Me}-$ dium (Figure 4c) or Leibovitz's L-15 Medium (Figure 4d) but are fixed in GA solution containing ruthenium red, on semithin sections a completely different histological pattern becomes visible as compared to samples fixed in conventional GA solution (Figure 4a,b). Ruthenium red label demonstrates that generated tubules are enclosed by an unexpected prominent basal lamina. Its extended scale is not recognized, when specimens are fixed in traditional GA solution.

Moreover, specimens generated in $\mathrm{CO}_{2}$ Independent Medium (Figure 4e) or Leibovitz's L-15 Medium (Figure 4f) but fixed in GA solution containing tannic acid exhibit a completely different cell pattern as compared to series fixed in conventional GA solution (Figure $4 \mathrm{a}, \mathrm{b}$ ) or GA solution containing ruthenium red (Figure 4c,d). Most impressive, fixation by GA solution contain- ing tannic acid depicts that in generated tubules two different types of cells are contained. Some segments of tubules show a homogenously composed bright or dark cell population $\left(\mathrm{T}^{\mathrm{B}}, \mathrm{T}^{\mathrm{D}}\right)$, while in other segments a heterogenously composed epithelium is developed consisting of dark and bright cells $\left(\mathrm{T}^{\mathrm{D} / \mathrm{B}}\right)$.

Thus, improved fixation of specimens in GA solution containing ruthenium red (Figure 4c,d) or tannic acid (Figure 4e,f) unmasks hidden morphological details at the basal lamina respectively illuminates an earlier not visible heterogenously composed cell population within the epithelium of generated tubules.

Finally, fixation by GA solution containing ruthenium red respectively tannic acid was performed for control also with embryonic, maturing and matured parenchyma of the kidney. However, neither the extended basal lamina nor the heterogenously composed epithelium consisting of bright and dark cells is observed in intact renal parenchyma. Since in none of the cases a coincidence is found, unveiled morphological details in generated tubules have to be ascribed to atypical cell development as it was recently described $[35,36]$.

\section{Disappointment meets Responsibility}

Due to the lack of donor organs and a limited period for dialysis an implantation of stem/progenitor cells into renal parenchyma still appears as an attractive alternative to cure acute and chronic renal failure in future $[37,38]$. However, earlier published data and here presented experiments put abundant expectations a bit more into a realistic perspective. In our sight much more intense research in the preclinical area must be performed in future, since the concept is limited by an up to date still ineffective implantation technique, a suboptimal seeding and a surprisingly small survival of stem/progenitor cells in diseased renal parenchyma [39].

Figure 4. Analysis of tubules generated in a POSI-4 polyester fleece and fixed by various methods. Semithin sections of specimens raised in ( $a, c, e) \mathrm{CO}_{2}$ Independent Medium or (b, d, f) Leibovitz's L-15 Medium demonstrate a simple epithelium with prismatic cells bordered by a lumen (arrow) and a basal lamina (asterisk). (a, b) Fixation of specimens in traditional GA solution followed by staining with Richardson exhibits that tubules contain a homogenous cell population. (c, d) Specimens fixed by GA solution containing ruthenium red show an unexpected thick basal lamina (asterisk) on tubules. $(c, d)$ Samples fixed by GA solution containing tannic acid illustrate tubules containing dark and bright cells $\left(\mathrm{T}^{\mathrm{D} / \mathrm{B}}\right)$ and tubules with only bright or dark cells $\left(T^{\mathrm{B}}, \mathrm{T}^{\mathrm{D}}\right)$.
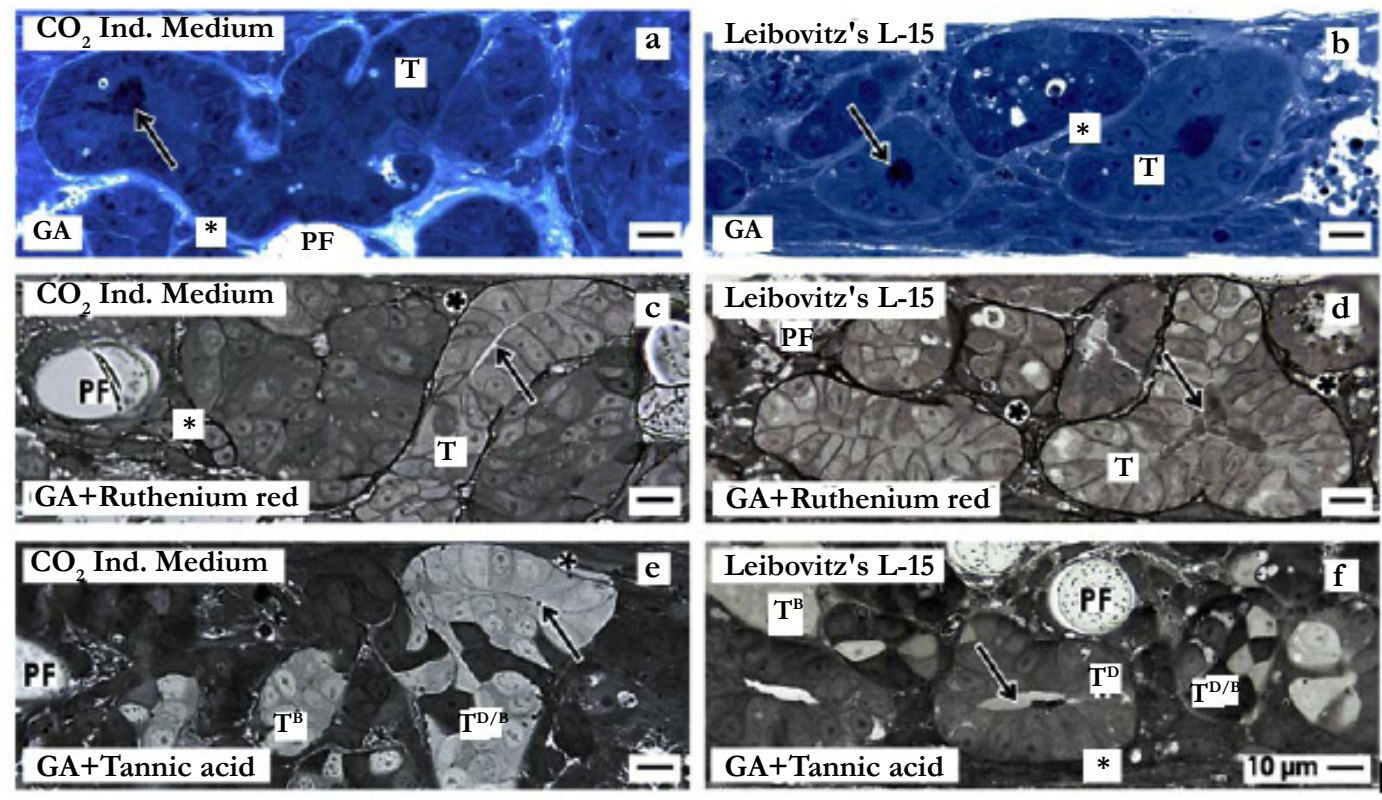

Minuth WW, Denk L (2015) Atypical Features in Regenerating Tubules Point to a Risk for Implantation of Renal Stem/Progenitor Cells. Int J Stem Cell Res Transplant 
Since application of innovative fixation for morphological analysis unexpectedly displays shocking results, pathologists must take a closer look at those cases in future. For example, specimens fixed by conventional GA solution suggest that in generated tubules an epithelium with an unremarkable cell population is present (Figure $4 a, b)$, while fixation of specimens in GA solution containing ruthenium red exhibits an altered basal lamina of generated tubules (Figure 4c,d). Further fixation in GA solution containing tannic acid illustrates that in tubules a heterogenously composed epithelium is present consisting of bright and dark cells (Figure $4 \mathrm{e}, \mathrm{f})$. A comparable epithelium was detected neither in embryonic, maturing nor in matured renal parenchyma [40, 41]. There is just one exception: the interstitial interface within the renal stem/progenitor cell niche exhibits label for ruthenium red and tannic acid [12-14]. This fact indicates that in generated tubules features of stem/progenitor cells are still contained. Finally, since an excess of vacuoles as an indicator for cytotoxicity is not observed, the bright and dark cell types do not reveal clear signs of programmed cell death (PCD) such as apoptosis or necroptosis [42]. Looking over all the intact appearance including integration within the tubule epithelium suggests that its formation is under control of initial differentiation. However, the illustrated bright and dark cell types are under influence of atypical development.

Regarding these disappointing result one could argue that atypical cell development (Figure 4c- $\mathrm{f}$ ) is caused as an effect of applied advanced culture conditions (Figure 1a-c). This aspect cannot be answered, since an implantation (Figure 1e,f) of here applied stem/progenitor cells has not been performed up to date. However, independent from that the here presented data clearly warn that in case of an implantation into an animal a risk for atypical cell development must be considered. In this coherence it makes no difference, whether atypical cell development is caused by here presented culture conditions or is evoked by harsh environment of diseased parenchyma after implantation. Irrespectively of whether it may be induced by culture conditions or evoked by the environment after implantation, it is shown that in renal stem/ progenitor cells principally both intact formation of tubules and a sleeping capacity for development of atypical cell and extracellular matrix is contained $[35,36]$.

\section{Conclusion}

Personal experiences demonstrate that there is a long and sometimes difficult way from the idea to its biomedical realization. When the project was started a decade ago, the primary focus of interest was directed to environmental adaption, when stem/progenitor cells are transferred during implantation from a beneficial culture medium to the inflammatory environment of diseased parenchyma. As an answer an artificial interstitium is under development to buffer the transition. Following this concept stem/ progenitor cells are loaded in a polyester fleece pad (Figure 1b) as a substitute for interstitial extracellular matrix. Under described conditions chemically defined culture mediacan be principally applied before (Figure 1d), during (Figure 1e) and after implantation (Figure 1f) via integrated afferent and efferent tubes. Although this strategy is laborious, it shed new light to recognize that a hidden cell biological risk in renal stem/progenitor cell development is contained. In a timely manner this risk became apparent, before an implantation in an animal was made. For that reason time in the laboratory is not lost but a gathered one.
The here presented data are based on the observation that conventional fixation by GA solution (Figure 4a,b) does not show all contained morphological details, while fixation by GA solution containing ruthenium red (Figure 4c,d) respectively tannic acid (Figure 4e,f) unmasks hidden morphological features. As a consequence, the present data show that advanced culture conditions in combination with innovative protocols for fixation are a huge opportunity for future research that allow to register an extended spectrum of morphological details. It is obvious that illustrated morphological data do not inform about all contained cell biological features. To obtain more solid information about illustrated pathological cell development, it is obvious that a bouquet of new antibodies and actual molecular techniques must be collated.

Finally, all parties involved in our laboratory must be happy that hard work over the past few years has paid off so that this unexpected risk becomes visible before stem/progenitor cells are implanted.

\section{Acknowledgements}

The authors thank the Institute of Molecular and Cellular Anatomy, University of Regensburg for financial support and technical assistance. Performed experiments are in accordance with the Animal Ethics Committee, University of Regensburg, Regensburg, Germany.

\section{Author Contributions}

WWM coordinated the experiments, analyzed specimens in fluorescence microscopy and interpreted results in microscopy, designed the figure presentations and wrote the manuscript. LD isolated renal stem/progenitor cells, prepared media and the artificial interstitium, performed perfusion culture and histochemical experiments, made special fixation, embedding, semithin sections, analysis in microscopy. Further she made all works dealing with figure presentation.

\section{References}

[1]. Caldas HC, Hayashi APC, Abbud-Filho M (2011) Repairing the chronic damaged kidney: the role of regenerative medicine. Transplantation Proceedings 43(10): 3573-3576.

[2]. Harari-Steinberg O, Metsuyanim S, Omer D, Gnatek Y, Gershon R, et al (2013) Identification of human nephron progenitors capable of generation of kidney structures and functional repair of chronic renal disease. EMBO Mol Med 5(10): 1556-1568.

[3]. Herrera M, Mirotsou M (2014) Stem cells: potential and challenges for kidney repair. Am J Physiol Renal Physiol 306(1): F12-F23

[4]. Caldas HC, de Paula Couto TA, Fernandes IM, Baptista MA, Kawasaki-Oyama RS, et al. (2015) Comparative effects of mesenchymal stem cell therapy in distinct stages of chronic renal failure. Clin Exp Nephrol 1-7.

[5]. Curtis LM, Chen S, Chen B, Agarwal A, Klug CA, et al. (2008) Contribution of intrarenal cells to cellular repair after acute kidney injury: subcapsular implantation technique. Am J Physiol Renal Physiol 295(1): F310-314.

[6]. Wang Y, He J, Pei X, Zhao W (2013) Systematic review and meta-analysis of mesenchymal stem/stromal cells therapy for impaired renal function in small animals. Nephrology 18(3): 201-208.

[7]. Shultz LD, Goodwin N, Ishikawa F, Hosur V, Lyons BL, et al. (2015) Subcapsular transplantation of tissue in the kidney. Cold Spring Harb Protoc 2014(7): 737-740.

[8]. Burst V, Pütsch F, Kubacki T, Völker LA, Bartram MP, et al. (2013) Survival and distribution of injected haematopoietic stem cells in acute kidney injury. Nephrol Dial Transplant 28(5): 1131-1139.

[9]. Minuth WW, Denk L (2014) Structural links between the renal stem/pro- 
genitor cell niche and the organ capsule. Histochem Cell Biol 141(5): 459471 .

[10]. Minuth WW, Denk L, Miess C, Glashauser A (2011) Peculiarities of the extracellular matrix in the interstitium of the renal stem/progenitor cell niche. Histochem Cell Biol 136(3): 321-334.

[11]. Minuth WW, Denk L (2012) Illustration of extensive extracellular matrix at the epithelial-mesenchymal interface within the renal stem/progenitor cell niche. BMC Clin Pathol 12(1): 16.

[12]. Minuth WW, Denk L (2013) The interstitial interface within the renal stem/ progenitor cell niche exhibits an unique microheterogeneous composition. Int J Mol Sci 14(7): 13657-13669.

[13]. Minuth WW, Denk L (2012) Cell projections and extracellular matrix cross the interstitial interface within the renal stem/progenitor cell niche: accidental, structural or functional cues? Nephron Exp Nephrology 122(3-4): 131-140.

[14]. Minuth WW, Denk L (2015) When morphogenetic proteins encounter special extracellular matrix and cell-cell connections at the interface of the renal stem/progenitor cell niche. Anat Cell Biol 48(1): 1-9.

[15]. Morigi M, Benigni A (2013) Mesenchymal stem cells and kidney repair. Nephrol Dial Transplant 28(4): 788-793.

[16]. Stine RR, Matunis EL (2013) Stem cell completion: finding balance in the niche. Trends Cell Biology 23(8): 357-364.

[17]. Bonventre JV, Yang L (2011) Cellular pathophysiology of ischemic acute kidney injury. J Clin Invest 121(11): 4210-4221.

[18]. Lee DW, Faubel S, Edelstein CL (2011) Cytokines in acute kidney injury (AKI). Clin Nephrol 76(3): 165-173.

[19]. Miyamoto T, Carrero JJ, Stenvinkel P (2011) Inflammation as a risk factor and target for therapy in chronic kidney disease. Curr Opinion Nephrol Hypertens 20(6): 662-668.

[20]. Chiang CK, Tanaka T, Nangaku M (2012) Dysregulated oxygen metabolism of the kidney by uremic toxins: review. J Ren Nutr 22(1): 77-80.

[21]. Roessger A, Denk L, Minuth WW (2009) Potential of stem/progenitor cell cultures within polyester fleeces to regenerate renal tubules. Biomaterials 30(22): 3723-3732.

[22]. Minuth WW, Denk L (2012) Interstitial interfaces show marked differences in regenerating tubules, matured tubules and the renal stem/progenitor cell niche. J Biomed Mater Res A 100(5): 1115-1125.

[23]. Gattazzo F, Urciuolo A, Bonaldo P (2014) Extracellular matrix: A dynamic microenvironment for stem cell niche. Biochim Biophys Acta 1840(8): 2509-2519.

[24]. Barak H, Huh SH, Chen S, Jeanpierre C, Martinovic J, et al. (2012) FGF9 and FGF20 maintain the stemness of nephron progenitors in mice and man. Dev Cell 22(6): 1191-1207.

[25]. Carroll TJ, Das A (2013) Defining the signals that constitute the nephron progenitor niche. J Am Soc Nephrol 24(6): 873-876.

[26]. Ahn SY, Kim Y, Kim ST, Swat W, Miner JH (2013) Scaffolding proteins DLG1 and CASK cooperate to maintain the nephron progenitor population during kidney development. J Am Soc Nephrol 24(7): 1127-1138.

[27]. Hilliard SA, Yao X, El-Dahr SS (2014) Mdm2 is required for maintenance of the nephrogenic niche. Dev Biol 387(1): 1-14.

[28]. Kopan R, Chen S, Little M (2014) Nephron progenitor cells: shifting the balance of self-renewal and differentiation. Curr Top Dev Biol 107: 293-331.

[29]. Minuth WW, Denk L, Gruber M (2013) Search for chemically defined culture medium to assist initial regeneration of diseased renal parenchyma after stem/progenitor cell implantation. Int J Stem Cell Res Transplant 1(02): 08-15.

[30]. Minuth WW, Denk L (2013) Initial steps to stabilize the microenvironment for implantation of stem/progenitor cells in diseased renal parenchyma. Transplant Technol 1(1): 2

[31]. Minuth WW, Denk L, Castrop H (2008) Generation of tubular superstructures by piling of renal stem/progenitor cells. Tissue Eng C Methods 14(1): 3-13.

[32]. Minuth WW, Denk L (2015) Advanced fixation for transmission electron microscopy unveils special extracellular matrix within the renal stem/progenitor cell niche. Methods Mol Biol 1212: 21-37.

[33]. Minuth WW, Denk L, Meese C, Rachel R, Roessger A (2009) Ultrastructural insights in the interface between generated renal tubules and a polyester interstitium. Langmuir 25(8): 4621-4627.

[34]. Luschnig S, Uv A (2014) Luminal matrices: an inside view on organ morphogenesis. Exp Cell Res 321(1): 64-70.

[35]. Minuth WW, Denk L (2014) Tannic acid label indicates abnormal cell development coinciding with regeneration of renal tubules. BMC Clin Pathol 14(1): 34.

[36]. Minuth WW, Denk L (2014) Detection of abnormal extracellular matrix in the interstitium of regenerating renal tubules. Int J Mol Sci 15(12): 2324023254 .

[37]. Winyard PJ, Price KL (2013) Experimental renal progenitor cells: repairing and creating kidneys? Pediatr Nephrol 29(4): 665-672.

[38]. de Almeida DC, Donizetti-Oliviera C, Barbosa-Costa P, Origassa CS, Camara NO (2013) In search of mechanisms associated with mesenchymal stem cell-based therapies for acute kidney injury. Clin Biochem Rev 34(3): 131-144.

[39]. Alagesan S, Griffin MD (2014) Autologous and allogenic mesenchymal stem cells in organ transplantation: what do we know about their safety and efficacy? Curr Opin Organ Transplant 19(1): 65-72.

[40]. Satlin LM, Matsumoto T, Schwartz GJ (1992) Postnatal maturation of rabbit collecting duct. III Peanut lectin-binding intercalated cells. Am J Physiol 262(2): F199-208.

[41]. Al-Aqati Q (2013) Cell biology of the intercalated cell in the kidney. FEBS Lett 587(13): 1911-1914.

[42]. Li X, Guo M, Shao Y (2013) Ultrastructural observations of programmed cell death during metanephric development in mouse. Microscopy Res Technique 76(5): 467-475. 